\title{
Reducing The Mobile Ip Binding Updates and The Packet Loss for The Repetitive Ip Stations During a Short Period of Time
}

\author{
Debabrata Sarddar \\ Department of E.T.C.E. \\ Jadavpur University
Suman Kumar Sikder
M.Tech in C.S.E
Jadavpur University
Shabnam Bandyopadhyay
Depart of C.S.E
Jadavpur University

\author{
Soumya Das \\ Department of E.T.C.E \\ Bengal Institute of Tech. \\ W.B.U.T \\ Sougata Chakraborty \\ M.Tech in C.S.E \\ Jadavpur University \\ Kalyan Kumar Das \\ M.Tech in C.S.E \\ Jadavpur University
}

\author{
Dipsikha Ganguly \\ Department of E.T.C.E. \\ B.P. Poddar I.M.T. W.B.U.T \\ Kunal Hui \\ M.Tech in C.S.E \\ Haldia Institute of Technology \\ Mrinal Kanti Naskar
Department of E.T.C.E
Jadavpur University
}

\begin{abstract}
LEO satellites have important advantages over geostationary satellite such as low power requirements, low propagation delay and more efficient spectrum delay. But, the handover management in LEO satellite becomes challenging for supporting global mobile communication. Here we propose a new method of introducing a location manager which will store the previous ip $\mathrm{s}$ depending on users choice and comparing the new ip address with the stored one and taking the decision whether to register or not. This method also reduces the binding updates and the packet loss during communication. It is the most useful process when the previous ip addresses are repeated for several times during a short span.
\end{abstract}

\section{Keywords}

Handover, location manager, satellite networks, binding update

\section{INTRODUCTION}

Satellite communication networks are utilized to co exist with terrestrial networks in order to provide global coverage to a heterogeneously distributed over population. A LEO satellite takes about 100 minutes to orbit the earth, which means that a single satellite is in view of ground equipment for only a few minutes [1]. As a consequence, a LEO satellite system must hand over between satellites to complete the transmission if a transmission takes more than the short time period that any one satellite is in view. In general, this can be accomplished by constantly

relaying signals between the satellite and various ground stations, or by communicating between the satellites themselves using "inter-satellite links" (ISLs) [1], [2]. LEO satellites are also designed to have more than one satellite in view from any spot on the earth at any given time, minimizing the possibility that the network will loose the transmission. Due to the fast-

flying satellites, LEO systems must incorporate complicated tracking and switching equipment to maintain consistent service coverage. In this paper, we focus on the handover management of satellite networks, specially the MIP which is a crucial design problem for supporting mobile communication services in the co-existing terrestrial and LEO satellite networks.

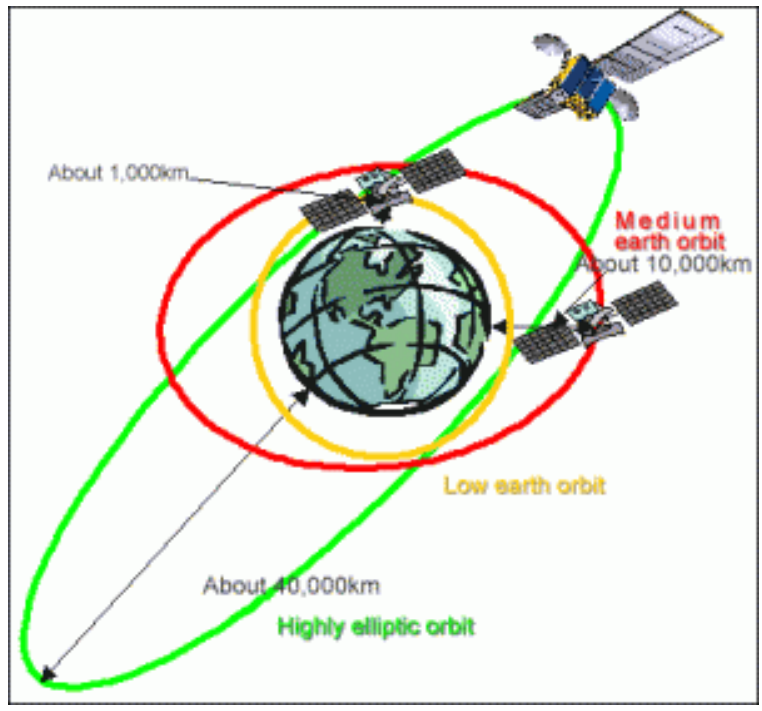

Handover:

In the term handover or handoff refers to the process of transferring an ongoing call or data session from one channel connected to the core network to another. In satellite communications it is the process of transferring satellite control responsibility from one earth station to another without loss or interruption of service. Handovers may degrade the system performance as an unsuccessful handover results call blocking and forced call termination. Forced call termination is less desirable than a new call blocking though both affect the performance of the system. A number of handover techniques have been proposed to solve this problem. One of the proposed models for handover management in satellite networks is mobile IP (MIP) [3]. When a mobile host moves from one point of attachment to another it enables a TCP connection to remain alive and to continue receiving packets. Although MIP is a widely used approach applied to satellite networks, it has some important drawbacks including high handover latency and high packet loss [4] [5].In our paper we have proposed an idea of reducing 
binding updates, handover latency and packet loss during handover. Our paper is structured as follows:

In the first section we have given a brief introduction related to LEO satellite and handover mechanisms. In section 2 we have discussed the related work regarding MIP network. In next section we have proposed our idea of introducing a location manager .In section $4 \& 5$ we have shown the simulation results and the conclusion and the future work regarding this paper.

\section{RELATED WORK}

Here we have described the related work and also our proposed work in the virtual environment of aysegul. The related works prior to our proposed algorithm have been discussed as follows:

\subsection{Mobile Mobility Management Related to MN:}

In low earth orbit (LEO) satellite network Mobility management is one of the key technologies. The aim of mobility management is to track where the subscribers are, allowing calls, SMS and other mobile phone services to be delivered to them. The main concern of mobility management is to locating MNs position in the network and guarantees a seamless data transmission upon change in nodes position. Mobility management basically contains two operations, namely binding update and data delivery. The binding update operation aims to associate reach ability identity (Reach.ID) and routing identity (Route.ID) of each node [6] [7], whereas, Reach.ID indicates a unique name of the node and is not subject to change. The Route.ID specifies the position of the node in the network and changes in response to node movement. When a mobile node changes its position, the Route.ID changes as well and the old binding is no longer valid. To update the binding, mobile nodes are requested to send their new Route.ID to the location directory (LD) [8]. The main problem of this procedure arises when LD is geographically too far from mobile nodes. In this case, binding update cost becomes very expensive, especially in a high mobility environment such as satellite networks [9]. Although a handover is a local process that concerns only the $\mathrm{MN}$, the old $\mathrm{AR}$, and the new AR, a binding update is a global process hat may affect other network elements in addition to the three adjacent entities. Route.ID can be used to locate the position of the $\mathrm{MN}$; therefore, no further operation is needed to transmit the data seamlessly. However, using Route.ID as the precise location of the MN requires frequent update of MNs registration even upon a slight movement of the nodes. Thus, the required update cost can be very huge [10]. On the other hand, when Route.ID is used to indicate location of the $\mathrm{MN}$ roughly, an additional operation called paging is needed to find precise position of the MN. However, the paging cost can be very high in case of wide paging areas. As a result, Route.ID has a significant importance on the mobility management cost. The role of the Route.ID should be chosen carefully according to mobility management issues of Underlying network.

\subsection{Mobility Management in Terrestrial Mobile Networks:} In terrestrial IP networks, IP addresses are designed for Route.IDs as well as Reach.IDs in higher layers. This causes an important problem for mobility management as $\mathrm{MN}$ can not be identified in the higher layers when its IP address changes after handover. The most dominant protocol among existing mobility management

Protocols are MIP that was proposed to solve this problem by using two different IP addresses for the two locations of MNs. First location is called as home network and identified by home address which serves as a Reach.ID. Second location is visiting network and identified by care of address (CoA) which functions as a Route.ID. In this protocol, locations of MNs are precisely managed by binding update for every handover occurrence. The details of MIP and its drawbacks will be discussed in the remainder of this section. Also, there are other mobility management protocols such as paging mobile IP (P-MIP) [11] and cellular IP [12] which are based on the principle of loose location management of idle nodes. In loose location management, location management is done for only idle nodes. When idle node becomes active, paging is usually used for locating the node in the network. Loose location management protocols have not covered here.

\subsection{Drawbacks of Mobility Management in LEO Satellite Networks:}

The drawbacks are mentioned as follows:

The most widely used protocol for mobility management over satellite networks is again mobile IP proposed by the Internet engineering task force (IETF) to handle mobility of Internet hosts for mobile data communications [13].The MIP enables IP host mobility without breaking the high level connection. It enables a TCP connection to remain alive and to continue receiving packets when an $\mathrm{MN}$ moves from allocation of channels, and updates its location in the HA database. The traffic flow of MIP is depicted in Fig. 1. When MN moves to a new domain, a location update is sent HA. Therefore, the HA is informed by the CoA of the MN. The packets from the $\mathrm{CN}$ to $\mathrm{MN}$ are encapsulated and forwarded to MN's current CoA. Then, these packets are encapsulated and delivered to upper layer protocols. Although MIP is a widely accepted concept, it has some drawbacks like

1. High Handover Latency: A MN needs to wait for completion of the steps, which are discovering the new CoA, registering the new CoA with the HA (binding update), and forwarding packets from the HA to the current CoA, before it can receive forwarded data from the previous point of attachment. Since the frequency of handover occurrences in LEO satellite networks is very high, a large number of binding update requests is likely to be generated in a single burst.

2. High packet lost rate is another drawback omit: During the HA registration period, some or all of the packets directed to the MN's old CoA will be lost because the old point of attachment does not know the new point of attachment of the $\mathrm{MN}$ so that it cannot communicate with the $\mathrm{MN}$ during this period.

3. Inefficient routing path: since large amount of data is routed to the HA, and then tunneled to the MN. This may decrease the scalability issues as the number of MNs managed by a HA increases.

4. Network security solution: The MIP conflicts with network security solutions such as ingress filtering and firewalls. It is hard to duplicate HA to various locations to increase survivability and manageability since HA must reside in MN's home network. Therefore, this model needs some modifications to be applicable to internet infrastructure.

Hence we have tried to solve these problems in our proposed work.

\section{PROPOSED WORK}

In our proposed work we have discussed about a new location manager which will reduce the drawback of handover latency of the MOBILE IP network. In a mobile IP network MN needs to wait for the completion of the steps which are discovering the new CoA, registering the new CoA with the Home Agent, and forwarding packets from HA to current 
CoA before it can receive forwarded data from the previous point of attachment. Here, we have seen that every time the satellite needs to register for the new IP and to forward packets. In our proposed location manager it will store the IP addresses for a limited time and if the satellite goes to the previous footprint

Again, it has no need to register; the previous IP address will be assigned to it. This will reduce the binding update and also the handover latency for the Mobile IP network. It is very much effective for the repetitive IP stations during a short period of time.

The location manager is also designed to reduce the packet loss of the network. In a mobile IP network during sending the packets if the acknowledgement is lost, it will send the previous data again and if it is sent they it will erase the previous data. Thus it will drastically reduce the packet loss in Mobile IP Handover.

\subsection{Algorithm:}

1. Location manager is activated.

2. While the mobile nodes changes its position it will store the IP addresses the new network.

3. For the next change in mobile IP address the address will be matched to the previous stored addresses, if it matches, no need to register again

Else, register the new IP address of the satellite.

The addresses will be stored up to $\mathrm{n}$ matches with the IP addresses $(n>0)$. The handover will be effective with the reducing values of $n$.

Now for the packet loss reduction after identifying the old imp address or registering the new one, the packets will be forwarded. It will check the acknowledgement after sending every packet and if acknowledgement $\mathrm{s}$ received then proceeds to the next

Else, resend it.

Thus this will also reduce the packet loss of the network.

Though it's an effective method, it will increase the time during the matching of data and the time will reduce with the value of $\mathrm{n}$ and also if the same IP address repeats in a short time period.

\subsection{Flow Chart:}

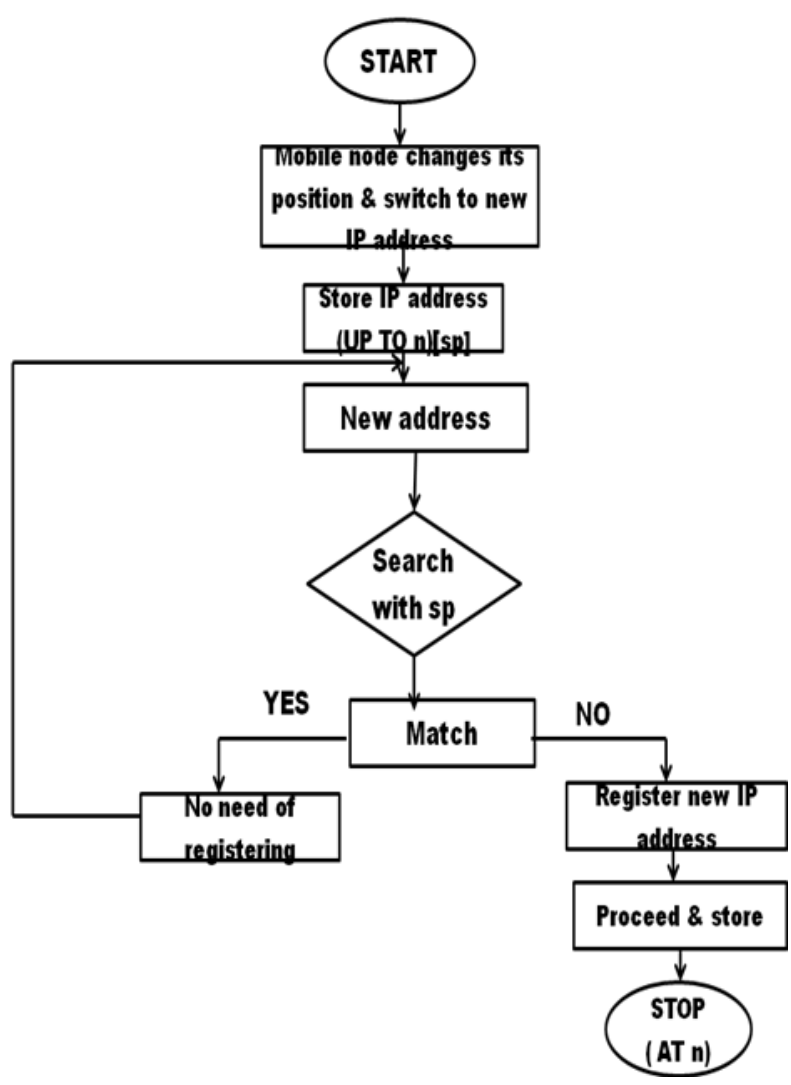

Fig1.

Then this loop will again go back to sp to store the data i.e. the addresses. Number of data to be stored i.e. $\mathrm{n}$ is basically user defined.

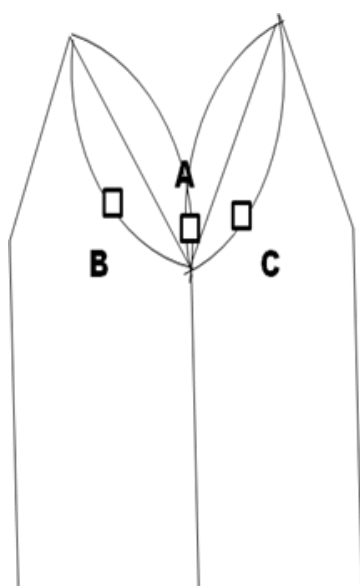

When the satellite A moves to $B$ and $C$ very frequently it has no need to register for the second time after the very first registration as the ip address is already there. Thus it reduces the binding updates of the satellite during handover.

Fig2 


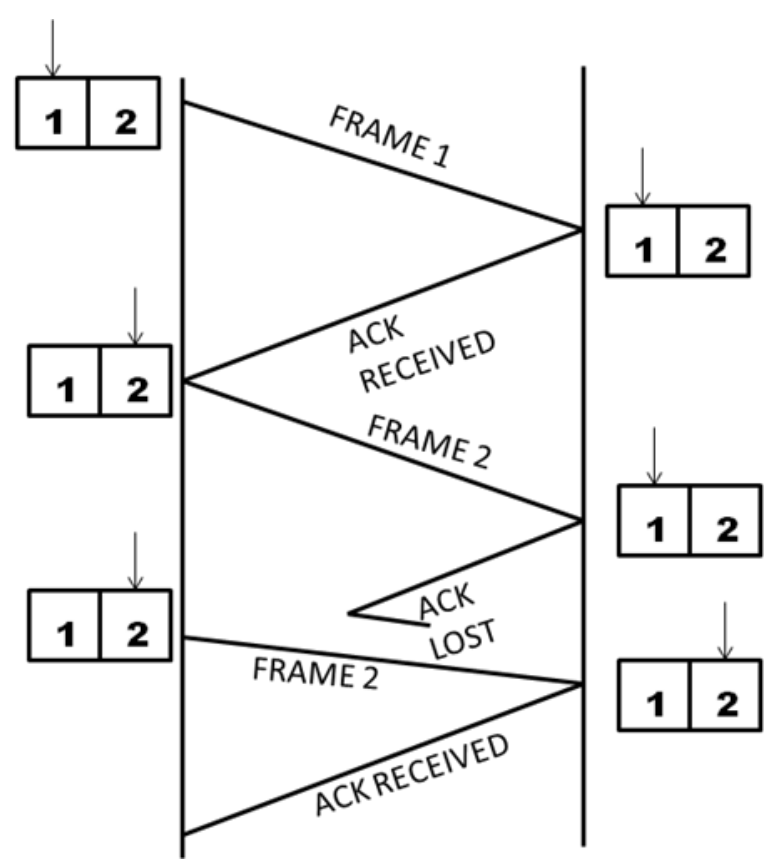

Fig3

In fig 3 we have shown the less data loss criteria of our proposed model. As we have said earlier that if the acknowledgement is lost the previous frame will be resend has been shown here schematically.

\section{SIMULATION RESULTS}

In the simulation part we have compared our proposed algorithm with the existing procedure related to the handover of the Mobile IP network. We have basically done three simulations here. First one is based on handover latency, second one is call blocking probability and the third one is the packet loss the results have been shown as follows:

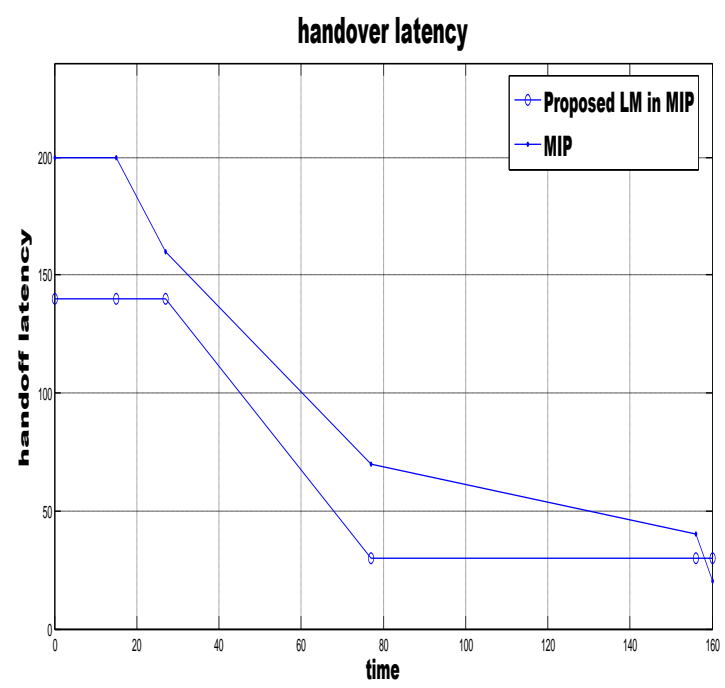

Fig4.handover latency

The above fig 4 is the simulation result of handover latency for MIP without applying our proposed algorithm and as well as after applying We have theoretically with the algorithm showed that the handover latency will be less in our proposed method now, here by simulation and Comparing both the waveforms we can conclude that introduction of the location manager in MIP will reduce the handover latency.

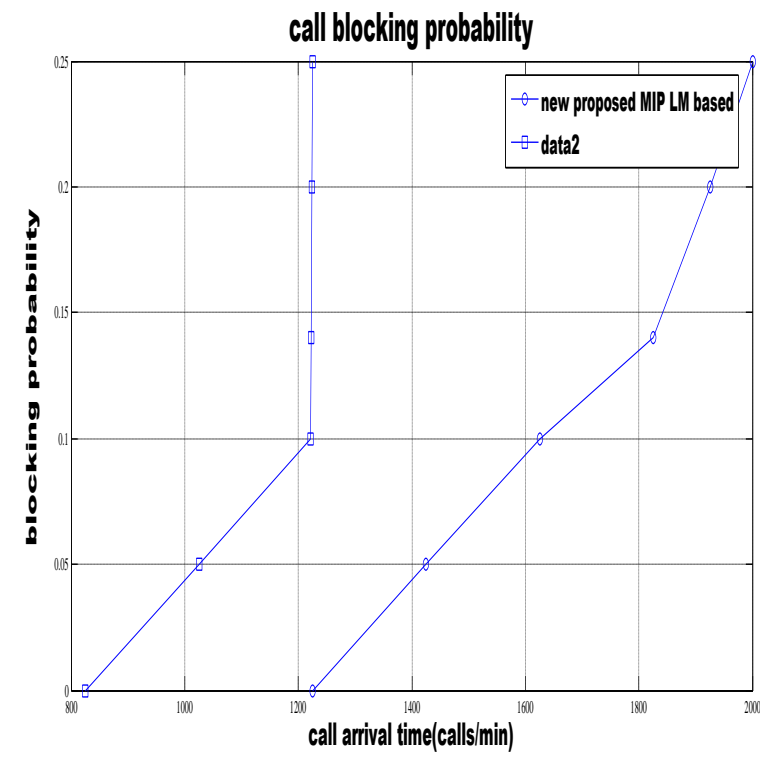

Fig5.call blocking probability

The above fig5 is the simulation result of both MIP without applying our proposed algorithm and after applying. Comparing both the waveforms we say conclude that in our process to some extent call blocking will be less.

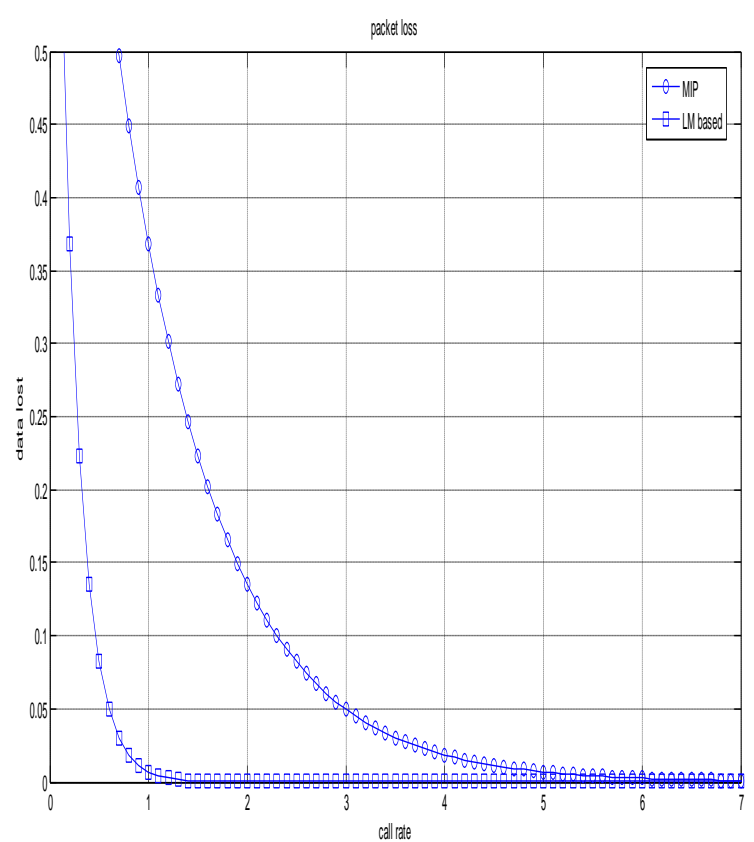

Fig6.packet loss

From the simulation results we can find it out that if LM based MIP is used it will reduce the data loss or the packet loss during transmission as it sends the data again after sending if it finds that the acknowledgement is lost. 


\section{CONCLUSION}

In this paper we have discussed the satellite handover mainly related to Mobile IP network. We found the drawbacks of these concepts like high handover latency, packet or data loss, inefficient routing and some others. In our proposed work we have defined a new location manager and also proposed an algorithm regarding its operation by using which we have shown that handover latency, binding updates, packet loss during transmission can be easily reduced. Hence we can conclude that our proposed work is better than the regular one. The future work regarding this can be done is to remodeling the LM (Location Manager) by reducing the space occupied by the stored address content.

\section{REFERENCES}

[1] Telecommunications, vol. 4, Wiley, 2002, pp. 2111-2122.

[2] L. Strand, "Linux mobile IPv6 HOWTO," Apr. 2004.

[3] Satellite Mobility Pattern Scheme for Centrical and Seamless Handover Management in LEO SatelliteNetworksAys seg"ul T"uys"uz and Fatih Alag"oz

[4] H. Uzunalioglu, I. F. Akyildiz, Y. Yesha, and W. Yen, "Footprint handover

rerouting protocol for low earth orbit satellite networks," WirelessNetworks, vol. 5, no. 5,

pp. 327-337, 1999

[5] A handoff management for Next Generation Wireless Systems By Joydeep Banerjee D Sarddar, S.K. Saha, M.K. Naskar, T.Jana, U. Biswas

[6] J. T. Malinen and C.Williams, "Micromobility taxonomy," Internet Draft,IETF, Nov. 2001

[7] P. Bhagwat, C. Perkins, and S. Tripathi, "Network layer mobility: An architecture and survey," IEEE Pers. Commun., vol. 3, no. 3, pp. 54-64, June1996.

[8] A. T. Campbell, J. Gomez, S. Kim, Z. Turanyi, C.Y. Wan, and A. Valko, Comparison of IP micromobility protocols," IEEE Wireless Commun. Mag., vol. 9, no. 1, Feb. 2002.

[9] H. Tsunoda, K. Ohta, N. Kato, and Y. Nemoto, "Supporting IP/LEO satellite networks by handover-independent IP mobility management," IEEE J.Select. Areas Commun., vol. 22, no. 2, pp. 300-307, 2004.

[10] X. Zhang, J. G. Castellanos, and A. T. Campbell, "Design and performance of mobile IP paging," ACM Mobile Networks and Appl., vol. 7, no. 2, Mar.2002.

[11]A. G. Valko, "Cellular IP-a new approach to Internet host mobility," ACMComputer Commun. Rev., Jan. 1999.

[12] C. Perkins, "IP mobility support," RFC 2002, IEFT, Oct. 1996, available at http://www.ietf.org/rfc/rfc2002.txt.
[13] K. Leung, D. Shell, W. Ivancic, D. Stewart, T. Bell, and B. Kachmar, "Application of mobile-IP to space and aeronautical networks," IEEE Aerosp. Electron. Syst. Mag., vol. 16, no. 12, pp. 13-18, Dec. 2001

[14] M. Atiquzzaman, S. Fu, and W. Ivancic, "TraSH$\mathrm{SN}$ : A transport layer seamless handoff scheme for space networks," in Proc. ESTC 2004, Palo Alto, CA, June 2004

[15]Y. H. Kwon and D. K. Sung, "Analysis of handover characteristics in shadowed LEO satellite communication networks," Int. J. Satellite Commun.,vol. 19, no. 6, pp. 581-600, Nov. 2001.

[16] R. Droms, "Dynamic host configuration protocol (DHCP),” RFC 2131,IETF, Mar. 1997.

[17] R. Droms, "Stateless dynamic host configuration protocol (DHCP) service for IPv6," RFC 3736 , IETF, Apr. 2004

[18] S. Thomson and T. Narten, "IPv6 stateless address autoconfiguration,"RFC 2462, IETF, Dec. 1998

[19] Minimization of Call Blocking Probability using Auxiliary Stations by D.sarddar,shubhajeet chatterjee,mandovi mukherjee,ijcaonline.org, International Journal of Computer Applications (0975 - 8887) Volume 25-No.7, July 2011

[20]Debabrata Sarddar, Soumya Das,Dipsikha Ganguly,Sougata Chakraborty,M.k.Naskar, A New Method for Fast and Low Cost Handover in Leo Satellites(International Journal of Computer Applications (0975 - 8887) Volume 37- No.7, January 2012) http://www.ijcaonline.org/archives/volume37/num ber7/4622-6631

[21] Debabrata Sarddar, Soumya Das,Dipsikha Ganguly,Sougata Chakraborty,M.K.Naskar, A New Method for Controlling Mobility Management Cost of PatHO- LEO Satellite and Mobile IP Network (International Journal of Computer Applications (0975 8887)Volume37-No.7,January2012 http://www.ijcaonline.org/archives/volume37/number7/4 $\underline{621-6630}$

\section{AUTHORS PROFILE}

Debabrata Sarddar is currently pursuing his $\mathrm{PhD}$ at Jadavpur University. He completed his M.Tech in Computer Science \& Engineering from DAVV, Indore in 2006, and his B.Tech in Computer Science \& Engineering from Regional Engineering College, Durgapur in 2001. His research interest includes wireless and mobile communication.

Soumya Das, son of Mr. Subrata Das and Mrs. Swapna Das, currently pursuing his B.Tech in Electronics \& Communication Engg. at Bengal Institute of Technology under West Bengal University of Technology. His research interest includes mobile communication \& satellite communication.

Dipsikha Ganguly, currently pursuing B.Tech in Electronics \& Communication engineering at B.P.Poddar Institute of Management \& Technology under West Bengal University of 
Technology. Her research interest includes wireless communication \& satellite communication. Email:

Suman Kumar Sikder completed his B.Tech in CSE from Murshidabad College of Engineering \& Technology and M.Tech in Computer Science \& Engineering from Jadavpur University in 2011. His research interest includes wireless sensor networks and wireless communication systems.

Sougata Chakraborty is presently an employee of IBM (Kolkata). He has completed his B.Tech in IT from Murshidabad College of Engineering and Technology and M.Tech in Computer Science \& Engineering from Jadavpur University in 2011. His research interest includes wireless sensor networks and wireless communication systems.

Kunal Hui is presently an employee of Cognizant (Kolkata). $\mathrm{He}$ has completed his B.Tech in Computer Science \& Engineering from Murshidabad College of Engineering and Technology (2004-2008) and M.Tech in Computer Science \& Engineering from Haldia Institute of Technology (20092011). His research interest includes wireless sensor networks and wireless communication systems.

Shabnam Bandyopadhyay is presently an employee of Cognizant (Kolkata). She has completed her B.Tech in Electronics and Communication Engineering from Meghnad Saha Institute of Technology and pursuing M.Tech degree in Computer Science \& Engineering at Jadavpur University Her research interest includes wireless sensor networks and wireless communication systems

Kalyan Kumar Das completed his BE degree in Computer Science and Engineering from REC (NIT), Durgapur in 2002 and M.Tech. in Computer Technology from Jadavpur University in 2011. His research interest includes wireless sensor networks and wireless communication systems.

Mrinal Kanti Naskar received his B.Tech. (Hons) and M.Tech degrees from E\&ECE Department, IIT Kharagpur, India in 1987 and 1989 respectively and Ph.D. from Jadavpur University, India in 2006.. He served as a faculty member in NIT, Jamshedpur and NIT, Durgapur during 1991-1996 and 1996-1999 respectively. Currently, he is a professor in the Department of Electronics and Tele-Communication Engineering, Jadavpur University, Kolkata, India where he is in charge of the Advanced Digital and Embedded Systems Lab. His research interests include ad-hoc networks, optical networks, wireless sensor networks, wireless and mobile networks and embedded systems. He is an author/co-author of the several published/accepted articles in WDM optical networking field that include "Adaptive Dynamic Wavelength Routing for WDM Optical Networks" [WOCN,2006], "A Heuristic Solution to SADM minimization for Static Traffic Grooming in WDM uni-directional Ring Networks" [Photonic Network Communication, 2006], "Genetic Evolutionary Approach for Static Traffic Grooming to SONET over WDM Optical Networks" [Computer Communication, Elsevier, 2007], and "Genetic Evolutionary Algorithm for Optimal Allocation of Wavelength Converters in WDM Optical Networks" [Photonic Network Communications,2008]. Fast Handoff Implementation by using Curve Fitting Equation With Help of GPS. 